\title{
Inhibition of MAPT enhances the effect of bexarotene and attenuates the damage after traumatic brain injury using in vivo and in vitro experiments
}

\author{
Haihai Dong*, Haitao Wang*, Liang Wang \\ Anqiu People's Hospital, Weifang, China \\ *Haihai Dong and Haitao Wang contributed equally in this work.
}

\begin{abstract}
Traumatic brain injury (TBI) is the leading cause of death and disability around the world in all age groups. The primary injury of $T B /$ is exacerbated by secondary injury, leading to an increased inflammatory response, cell death and even impairment of neurological function. Bexarotene has been found to improve neurological function in mice in an ApoE-dependent manner, but the detailed mechanism is not fully clear. Upregulated expression of MAPT has been found in mouse models after TBI; therefore, we hypothesized that inhibition of MAPT might contribute to the effects of bexarotene treatment in TBI models. Herein, we found that inhibition of MAPT enhanced the effects of bexarotene in increasing cellular viability and restoring brain function, and expression of anti-oxidative and anti-apoptotic molecules were elevated in response to inhibition of MAPT. These effects might be mediated by activation of the Nrf2/HO-1 signalling pathway and inhibition of the MAPK/NF-кB signalling pathway. Thus, we concluded that inhibition of MAPT might represent a novel treatment target for TBI.
\end{abstract}

Key words: MAPT, bexarotene, traumatic brain injury, Nrf2, oxidative stress.

\section{Introduction}

Traumatic brain injury (TBI) is a major healthrelated problem and affects 2.8 million people in the US each year, causing $30 \%$ of all injury-related deaths [26]. Characteristic consequences of TBI include neural damage, cell death and disruption of normal brain function, and TBI survivors frequently suffer emotional changes [1]. Bexarotene, a selective inhibitor of the retinoid $X$ receptor (RXR), has been found to improve spatial memory in the mice model after TBI via inhibition of cell apoptosis [33]. However, the detailed mechanism is not fully understood. MAPT is continuously expressed in neurons, especially in non-myelinated axons of cortical interneurons located in the grey matter [27]. Previous findings indicate that release of MAPT is an indicator of neurotrauma because the increased expression of MAPT has been observed in response to trauma $[19,20]$. Therefore, we hypothesized that inhibiting MAPT expression might enhance the treatment effects of bexarotene. In this study, we found that inhibition of MAPT enhanced cell viability and brain function of mice after treatment 
with bexarotene. We further observed that oxidative stress was attenuated after inhibition of MAPT, and expression of oxidative stress response-related molecules were increased. These effects might be mediated by activation of nuclear factor erythroid 2-related factor 2 (Nrf2)/hemeoxygenase-1 (HO-1) and inhibition of $\mathrm{MAPK} /$ nuclear factor $\kappa \mathrm{B}(\mathrm{NF}-\kappa \mathrm{B})$ signalling pathways.

\section{Material and methods Reagents and method}

H-DMEM (11965092), FBS (16140071) and Lipofectamine 3000 transfection reagent (L3000001) were purchased from Gibco. Xbal (R0145S), EcoRI (R3101S), BsmBI (R0580S) and Quick ligase (M2200S) were purchased from NEB. G418 (G8160), puromycin (P8230), MTT reagent (M8180), RIPA (R0010) and BCA kit (PC0020) were purchased from Solarbio. Bexarotene (200499) was purchased from Sigma. Anti-Tau (ab32057), p38 (phospho Y182) (ab47363), p38 (ab170099), AP1 (ab21981), Keap1 (ab118285), Nrf2 (ab137550), Heme

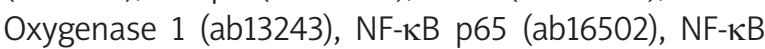
p65 (phospho S536) (ab86299), NQO1 (ab28947), GCLC (ab190685), glutathione reductase (ab124995), Bcl-2 (ab182858), and Bax (ab32503) antibodies, as well as Cellular ROS Assay (ab186029), Nitric Oxide Synthase Activity Assay Kit (ab211084), Hydrogen Peroxide Assay Kit (ab102500) and GSH/GSSG Ratio Detection Assay Kit (ab138881) were purchased from Abcam.

\section{Vector construction and cell model construction}

Full length MAPT cDNA was replicated using the following primers: Forward: 5'-GGCTCATTAGGCAACATCC-3', Reverse: 5'-AGCTGGGGCGAGTCTACCAT-3'. MAPT cDNA and pcDNA3.1 blank vector were digested with Xbal and EcoRI. Then, the pcDNA3.1-MAPT vector was transfected into cells using Lipofectamine 3000 transfection reagent for $48 \mathrm{~h}$ according to the protocol, and stable expressing pcDNA3.1-MAPT cells were selected using G418 at a concentration of $800 \mu \mathrm{g} / \mathrm{ml}$. A MAPT knockdown vector was constructed using the CRIPSR/Cas9 system. Briefly, the CRISPR blank vector was digested using BsmBI, and a pair of oligos were replicated with the following primer: Forward: 5'-CACCGGATCTCCGTGTGGGGCTGCG-3', Reverse: 5'-AACCGCAGCCCCACACGGAGATCCGGTGC-3'. The pair of primers and vector were ligated with Quick Ligase to construct the MAPT knockdown vector. The vector was firstly transfected into 293T cells to construct a lentivi- ral vector, and then H19-7 cells were infected with the lentivirus vector. Cells stably expressing MAPT knockdown were selected using $2 \mu \mathrm{g} / \mathrm{ml}$ puromycin.

\section{Cells and mouse model construction and grouping}

H19-7 (CRL-2526) and HEK293T cells (CRL-11268) were purchased from ATCC. Cells were cultured in H-DMEM supplemented with $10 \%$ fetal bovine serum (FBS) medium at a $34^{\circ} \mathrm{C}$ in a humidified $5 \% \mathrm{CO}_{2}$ atmosphere. Then, cells were divided into four groups: TBI (TC), TBI combined with bexarotene treatment (BT), $\mathrm{TB}$ combined with bexarotene treatment and MAPT overexpression (BO) and $\mathrm{TBI}$ combined with bexarotene treatment and MAPT inhibition (BI). In bexarotene treatment groups, cells were treated with $10 \mu \mathrm{M}$ bexarotene for $12 \mathrm{~h}$ according to a previous study [4]. Five MAPT overexpression mice, 5 MAPT knockdown mice and 10 normal C57/BL mice were purchased from the Academy of Military Medical Sciences. Mice were divided into four groups: TBI (TG), TBI combined with bexarotene treatment (TB), TBI combined with bexarotene treatment and MAPT overexpression (TO) and $\mathrm{TBI}$ combined with bexarotene treatment and MAPT inhibition ( $\mathrm{TI})$. Mice were kept in a $22-24^{\circ} \mathrm{C}$ atmosphere with water and food freely available, and mice in the bexarotene treatment group were treated with $5 \mathrm{mg} / \mathrm{kg}$ until subsequent experiments. To construct the TBI model, cells were seeded into a 6-well plate and scratched manually with a sterile plastic needle using a $9 \times 9$ square grid for $24 \mathrm{~h}$ according to a previous study [13]. The mice model of TBI was constructed as the previous study described [29]. Briefly, mice were anaesthetized with ketamine $(100 \mathrm{mg} / \mathrm{kg}) / x y l a z i n e$ (10 mg/kg) via the intraperitoneal injection, and then craniotomy was performed with a hand-held electrical drill (Dremel $10.8 \mathrm{~V}$ ). The bone flap was removed to expose the right parietal cortex, and the mice were placed in a stereotaxic device and subjected to a $1.5-\mathrm{mm}$ deep impact (velocity of $5 \mathrm{~m} / \mathrm{s}$ ) via a computer-controlled impactor device (LinMot-Talk 1100) (impactor diameter of $2 \mathrm{~mm}$ ). After successful $\mathrm{CCl}$, serum samples and brain tissues were collected after euthanasia to perform the subsequent experiments.

\section{Ethical statement}

Experiments on animal models were performed under the guideline of the Declaration of Helsinki and the principles and procedures outlined in the 
National Institutes of Health Guide for the Care and Use of Animals. Experiments on animal models were performed in Anqiu People's Hospital and were approved by the Health Animal Care and Use Committee of Anqiu People's Hospital.

\section{MTT assay}

MTT assay was performed according to the manufacturer's protocol. Briefly, cells were seeded into a 96-well plate at a concentration of $1 \times 10^{4}$ cells/ well, and cultured until a confluence of $70-80 \%$. Then, cells were grouped and treated as described in the previous section. After treatment, cells were incubated with MTT reagent at a concentration of $5 \mathrm{mg} / \mathrm{ml}$ for $4 \mathrm{~h}$. OD values were measured at $490 \mathrm{~nm}$ after incubation with $150 \mu \mathrm{LMSO}$, and cell viability was calculated using the following formula: viability rate $=\left(O D_{\text {Experiment }}-O D_{\text {Blank }}\right) /\left(O D_{\text {Control }}-O D_{\text {Blank }}\right)$.

\section{RNA extraction}

RNA extraction was performed according to the protocol of RNApure Tissue \& Cell Kit (CWBio, CW0584). Briefly, cells and brain tissues were lysed with lysis buffer, and after centrifuging at 12000 rpm for $5 \mathrm{~min}$, samples were incubated with ethanol and then loaded onto an absorption tube. After washing with washing buffer, the samples were eluted with elution buffer, and the RNA concentration was detected using a Nano drop 2000. RNA samples were store at $-80^{\circ} \mathrm{C}$ for subsequent experiments.

\section{Reverse transcription and $\mathrm{qPCR}$}

Reverse transcription and quantitative polymerase chain reaction ( $\mathrm{QPCR}$ ) were performed according to the protocol of UltraSYBR One Step RT-qPCR Kit (CWBio, CW2623). Briefly, the reaction buffer was mixed as recommended, and the reaction was performed using the following steps: reverse transcription at $45^{\circ} \mathrm{C}$ for $10 \mathrm{~min}$, predegeneration at $95^{\circ} \mathrm{C}$ for $5 \mathrm{~min}$, repeating of these steps for 40 cycles: degradation at $95^{\circ} \mathrm{C}$ for $10 \mathrm{~s}$ and extension at $60^{\circ} \mathrm{C}$ for $45 \mathrm{~s}$. The reaction was performed using the following primers: Nrf2: Forward: 5'-CTTGGCCTCAGTGATTCTGAAGTG-3', Reverse: 5'-CCTGAGATGGTGACAAGGGTT GTA-3'; HO-1: Forward: 5'-CAGGAGCTGCTGACCCATGA-3', Reverse: 5'-AGCAACTGTCGCCACCAGAA-3'; glutamate-cysteine ligase catalytic subunit gene (GCLC): Forward: 5'-GAAGTGGATGTGGACACCAGATG-3', Reverse: 5'-TTGTAGTCAGGATGGTTTGCGATAA-3'; NAD(P)H: quinone oxidoreductase 1 (NQO1): Forward: 5'-GGATTGGACCGAGCTGGAA-3', Reverse: 5'-AATTGCAGTGAAGATGAAGGCAAC-3'; Bcl-2: Forward: 5'-ATAACGGAGGCTGGGTAGGT-3', Reverse: 5'-TTTATTTCGCCGGCTCCACA-3'; Bax: Forward: 5'-GCCCTTTTGCTTCAGGGGATG-3', Reverse: 5'-CAGCTGCCACTCGGAAAAAG-3'. Each experiment was independently repeated three times and GAPDH was used as an internal control. Expression of each target gene was determined using the $2-\Delta \Delta \mathrm{Cq}$ method [12].

\section{Protein extraction and western blotting analysis}

Cells and tissues were lysed in lysis buffer (RIPA supplemented with protease inhibitor cocktail) and protein samples were collected after centrifugation at $12,000 \mathrm{rpm}$ for $10 \mathrm{~min}$. The concentration of protein samples were determined using bicinchoninic acid (BCA) assay. Then, $60 \mu \mathrm{g}$ protein samples were used to perform 10\% SDS-PAGE electrophoresis. After electrophoresis, proteins were transferred onto nitrocellulose membranes and then blocked in 5\% skim milk. Then, membranes were incubated with primary antibodies (1 : 1000) overnight at $4^{\circ} \mathrm{C}$ and secondary antibody $(1: 5000)$ at room temperature for $1 \mathrm{~h}$. The grey value of proteins was detected using chemiluminescent immunoassay, and GAPDH was used as an internal control.

\section{ELISA}

The enzyme-linked immunosorbent assay (ELISA) assay was performed according to the protocol. Briefly, cells and serum were added into each well of a 96-well plate, and incubated at $37^{\circ} \mathrm{C}$ for $90 \mathrm{~min}$, followed by incubation with target antibodies at $37^{\circ} \mathrm{C}$ for $60 \mathrm{~min}$. After washing with washing buffer, samples were incubated with $A B C$ working solution at $37^{\circ} \mathrm{C}$ for $30 \mathrm{~min}$. Samples were incubated with TMB agent for $25 \mathrm{~min}$ at $37^{\circ} \mathrm{C}$ followed by incubation with stop solution. Absorbance values were measured at $450 \mathrm{~nm}$ using a microplate reader (Multiskan FC, Thermo).

\section{Statistical analysis}

Data from each experiment are presented as the mean \pm SEM. Each experiment was independently repeated three times. One-way ANOVA was used to analyse differences between groups using SPSS 22.0 software. $P$-values $<0.05$ were considered statistically significant. 


\section{Results}

\section{Detection of cellular proliferation using MTT assay}

As shown in Figure $1 A$, the viability rate of $\mathrm{H} 19-7$ cells in the BT, BI and BO groups was $118.2 \pm 8.4$, $149.1 \pm 10.2$ and $94.2 \pm 7.7$, respectively. Cell viability in the BT group was significantly increased compared to the TC group $(p<0.05)$, and compared to the BT group, cell viability was significantly increased in the $\mathrm{BI}$ group $(p<0.05)$ and significantly decreased in the BO group $(p<0.05)$. The results demonstrate that bexarotene attenuates damage in brain tissues after TBI, and overexpression of MAPT enhances this effect.

\section{Detection of MAPT expression in each group in cell and mouse models}

As shown in Figure $1 \mathrm{~B}$ and $\mathrm{C}$, expression of MAPT in cell and mouse models was detected using western blotting analysis. Expression of MAPT in TC, BO and $\mathrm{Bl}$ of cell models without treatment of bexarotene was $0.71 \pm 0.06,0.89 \pm 0.07$ and $0.43 \pm 0.04$, respectively. Expression of MAPT in TG, TO and TI cell models without treatment of bexarotene was $0.63 \pm 0.05,0.91 \pm 0.08$ and $0.36 \pm 0.03$, respectively. These results indicate that MAPT overexpression and inhibition models were successfully constructed in cells and mice.

\section{Detection of target genes at the transcriptional level in cell and mouse models}

As shown in Figures 2 and 3, the expression of Nrf2, HO-1, GCLC, NQO1, Bcl-2 and Bax at the transcriptional level was detected using $\mathrm{QPCR}$. The expression of $\mathrm{Nrf2}$ in $\mathrm{TC}, \mathrm{BT}, \mathrm{BI}$ and $\mathrm{BO}$ groups of $\mathrm{H} 19-7$ cells was $0.81 \pm 0.06,0.96 \pm 0.07,1.34 \pm 0.10$ and $0.72 \pm 0.06$, respectively, and the expression in TG, TB, TI and TO mouse models was $1.05 \pm 0.08,1.25$ $\pm 0.10,1.57 \pm 0.12$ and $1.06 \pm 0.08$. In addition, the expression of HO- 1 in these groups of cells was 0.90 $\pm 0.07,1.17 \pm 0.09,1.52 \pm 0.12$ and $0.94 \pm 0.07$, respectively, and it was $0.96 \pm 0.07,1.24 \pm 0.10,1.61 \pm 0.12$ and $1.05 \pm 0.08$, respectively, in the mouse model. The expression of GCLC in these cell models was $0.52 \pm 0.04,0.73 \pm 0.06,1.14 \pm 0.09$ and $0.55 \pm 0.04$, respectively, and it was $0.81 \pm 0.06,0.97 \pm 0.07,1.43$
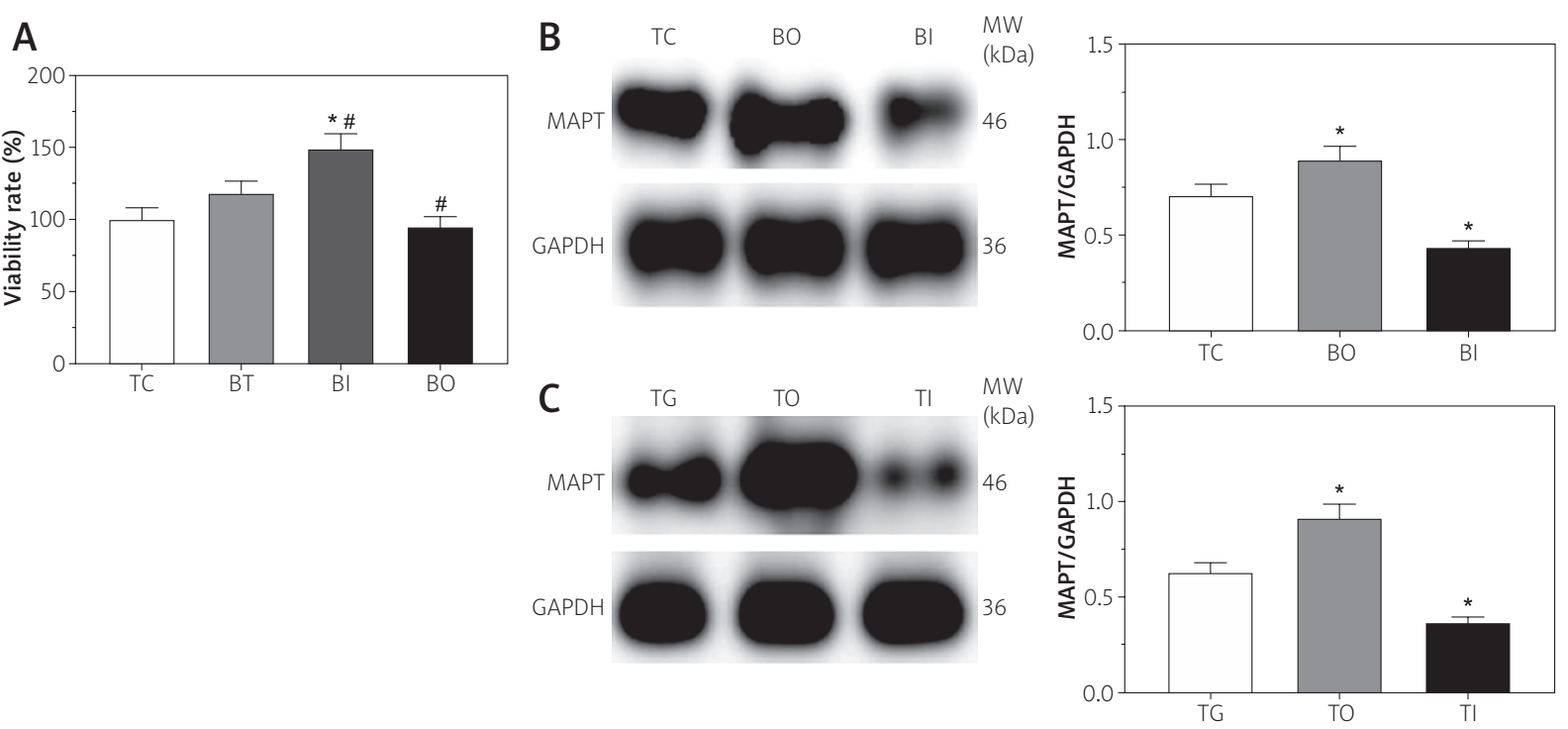

Fig. 1. The expression of MAPT in the cell and mice model without treatment of bexarotene. A) The viability rate of H19-7 cell in each group under treatment of bexarotene. B) The expression of MAPT in each group of H19-7 cell without treatment of bexarotene. $\mathrm{C}$ ) The expression of MAPT in each group of the mice model without treatment of bexarotene. ${ }^{*} p<0.05$ compared with the control group, ${ }^{*} p<0.05$ compared with the bexarotene treatment group. GAPDH was used as an internal control. Each experiment was repeated independently for three times. One-way ANOVA was used to compare the difference between groups. 
A

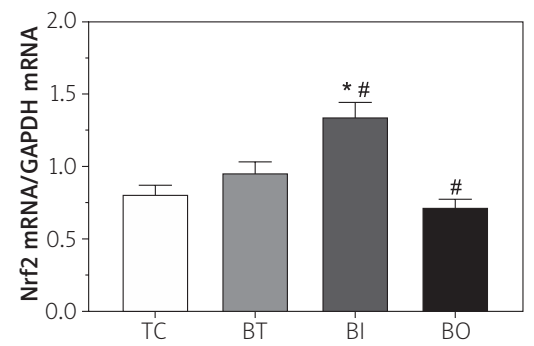

D

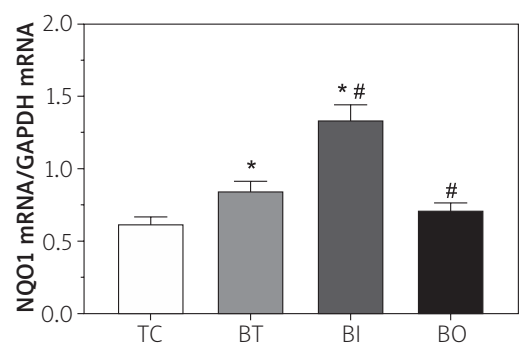

B

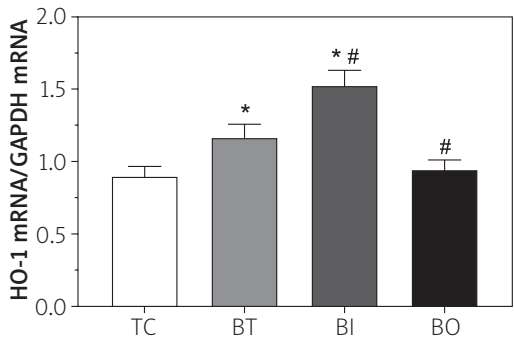

$\mathrm{E}$

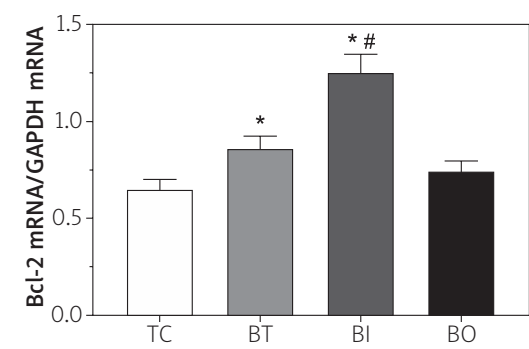

C

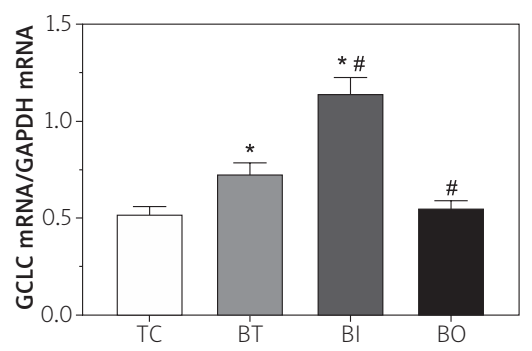

$\mathrm{F}$

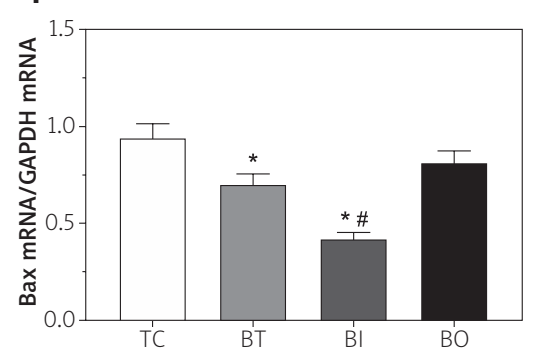

Fig. 2. Detection of oxidative response related factors at the transcription level in H19-7 cells using GPCR method. A) Expression of Nrf2 in each group of H19-7 cells. B) Expression of HO-1 in each group of H19-7 cells. C) Expression of GCLC in each group of H19-7 cells. D) Expression of NQO1 in each group of H19-7 cells. E) Expression of Bcl-2 in each group of H19-7 cells. F) Expression of Bax in each group of H19-7 cells. ${ }^{*} p<0.05$ compared with the control group, ${ }^{\#} p<0.05$ compared with the bexarotene treatment group. GAPDH was used as an internal control. Each experiment was repeated independently for three times. One-way ANOVA was used to compare the difference between groups.

A

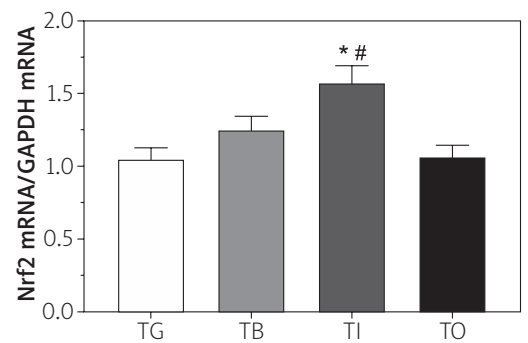

D

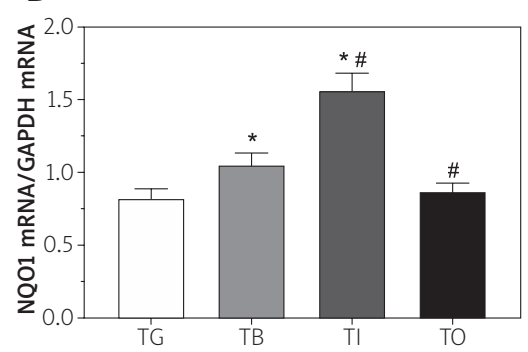

B

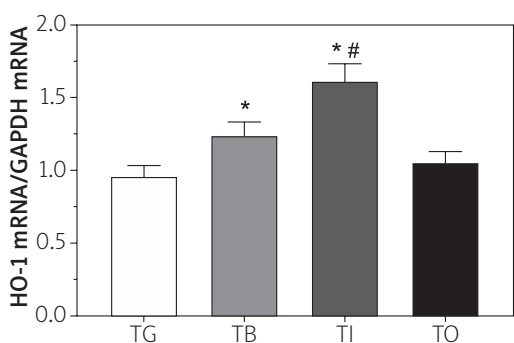

$\mathrm{E}$

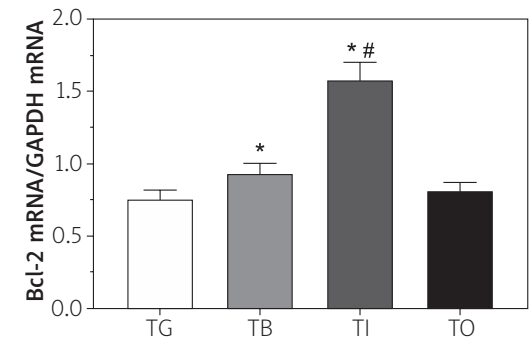

C

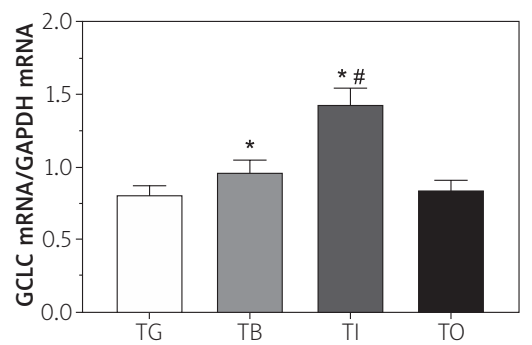

$\mathrm{F}$

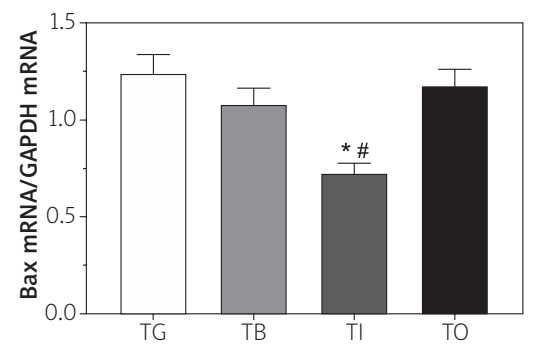

Fig. 3. Detection of oxidative response related factors at the transcription level in the mice model using qPCR method. A) Expression of Nrf2 in each group of the mice model. B) Expression of HO-1 in each group of the mice model. C) Expression of GCLC in each group of the mice model. D) Expression of NQO1 in each group of the mice model. E) Expression of Bcl-2 in each group of the mice model. F) Expression of Bax in each group of the mice model. ${ }^{*} p<0.05$ compared with the control group, ${ }^{*} p<0.05$ compared with the bexarotene treatment group. GAPDH was used as an internal control. Each experiment was repeated independently for three times. One-way ANOVA was used to compare the difference between groups. 
\pm 0.11 and $0.84 \pm 0.06$, respectively in each mouse model. The expression of NQO1 in these cell models was $0.62 \pm 0.05,0.85 \pm 0.07,1.34 \pm 0.10$ and 0.71 \pm 0.05 , respectively, and it was $0.82 \pm 0.06,1.05$ $\pm 0.08,1.56 \pm 0.12$ and $0.86 \pm 0.07$, respectively, in the mouse model. The expression of $\mathrm{Bcl}-2$ in each group of cells was $0.65 \pm 0.05,0.86 \pm 0.07,1.25 \pm 0.10$ and $0.74 \pm 0.06$, respectively, and it was $0.76 \pm 0.06$, $0.93 \pm 0.07,1.58 \pm 0.12$ and $0.81 \pm 0.06$, respectively in each group of mice. Expression of Bax in each group of cells was $0.94 \pm 0.07,0.70 \pm 0.05,0.42 \pm 0.03$ and $0.81 \pm 0.06$, respectively, and it was $1.24 \pm 0.10,1.08$ $\pm 0.08,0.72 \pm 0.06$ and $1.17 \pm 0.09$, respectively, in each group of mice. These results demonstrate that bexarotene treatment increased the expression levels of Nrf2/HO-1 and glutathione metabolism related enzymes, while inhibiting the apoptosis-related factors at the transcriptional level, suggesting that overexpression of MAPT might enhance the effect of bexarotene.

\section{Detection of target genes expression at the protein level in cell and mouse models}

As shown in Figures 4 and 5, the expression of each target protein in the cell and mice model was detected using western blotting analysis. Briefly, the expression of MAPT in TC, BT, BI and BO groups of $\mathrm{H} 19-7$ cells was $0.35 \pm 0.03,0.51 \pm 0.04,0.72 \pm 0.06$ and $0.05 \pm 0.01$, respectively, and it was $0.85 \pm 0.07$, $1.34 \pm 0.11,1.84 \pm 0.15$ and $0.84 \pm 0.07$ in TG, TB, TI and TO groups of mice, respectively. The expression of NQO1 in each group of cells was $0.18 \pm 0.02,0.65$ $\pm 0.05,1.06 \pm 0.09$ and $0.40 \pm 0.03$, respectively, and it was $1.27 \pm 0.11,1.97 \pm 0.16,2.54 \pm 0.21$ and $1.33 \pm 0.11$ in each group of mice, respectively. The expression of GCLC in each group of cells was $0.68 \pm 0.06$, $1.12 \pm 0.09,1.71 \pm 0.14$ and $0.72 \pm 0.06$, respectively, and it was $0.36 \pm 0.03,1.07 \pm 0.09,2.00 \pm 0.17$ and $0.92 \pm 0.08$ in each group of mice, respectively. The expression of glutathione-disulfide reductase (GSR) in each group of cells was $0.64 \pm 0.05,0.82 \pm 0.08$, $1.05 \pm 0.09$ and $0.59 \pm 0.05$, respectively, and it was $0.74 \pm 0.06,1.31 \pm 0.11,1.75 \pm 0.15$ and $0.48 \pm 0.04$ in each group of mice, respectively. The expression of $\mathrm{Bcl}-2$ in each group of cells was $0.66 \pm 0.05,0.73$ $\pm 0.06,0.83 \pm 0.07$ and $0.79 \pm 0.07$, respectively, and it was $0.24 \pm 0.02,0.71 \pm 0.06,0.86 \pm 0.07$ and 0.49 \pm 0.04 in corresponding mice. The expression of Bax in each group of cells was $1.80 \pm 0.15,1.07 \pm 0.09$, $0.52 \pm 0.04$ and $0.96 \pm 0.08$, respectively, and it was $1.32 \pm 0.11,0.94 \pm 0.08,0.49 \pm 0.04$ and $1.19 \pm 0.10$ in corresponding mice. These results demonstrate that bexarotene treatment combined with overexpression of MAPT significantly increased the expression of glutathione synthesis related enzymes and inhibited apoptosis, exerting a protective cellular effect.
A

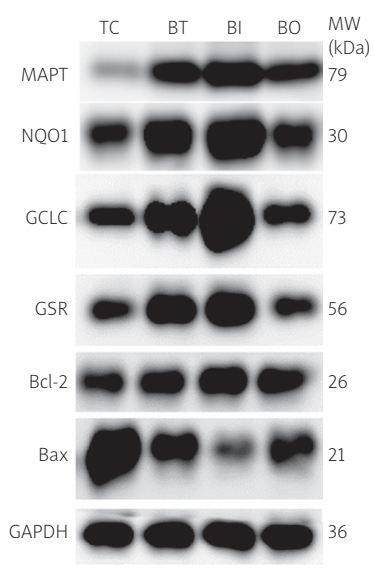

B
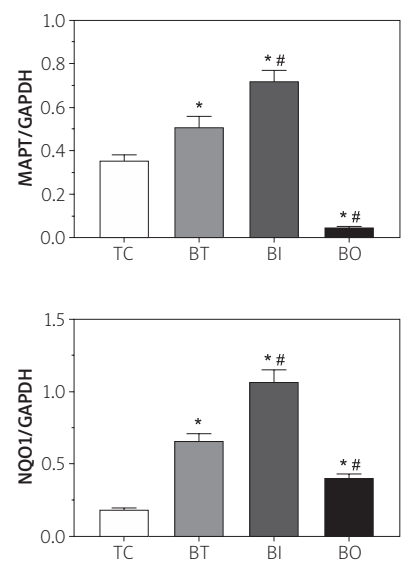
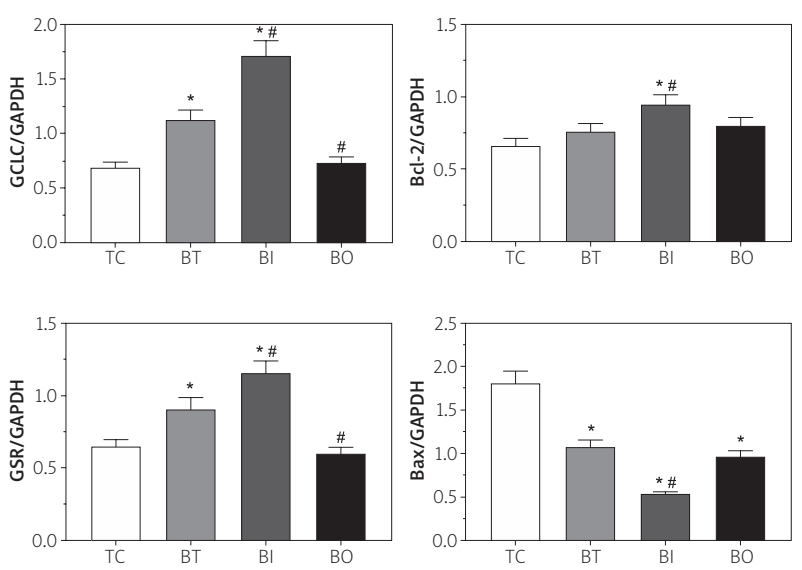

Fig. 4. Detection of glutathione synthesis and cellular apoptosis related enzymes expression in H19-7 cells using Western blotting analysis. A) Expression of glutathione synthesis and cellular apoptosis related enzymes. B) Quantitative analysis of each target proteins. ${ }^{*} p<0.05$ compared with the control group, ${ }^{\#} p<0.05$ compared with the bexarotene treatment group. Each experiment was repeated independently for three times. One-way ANOVA was used to compare the difference between groups. 

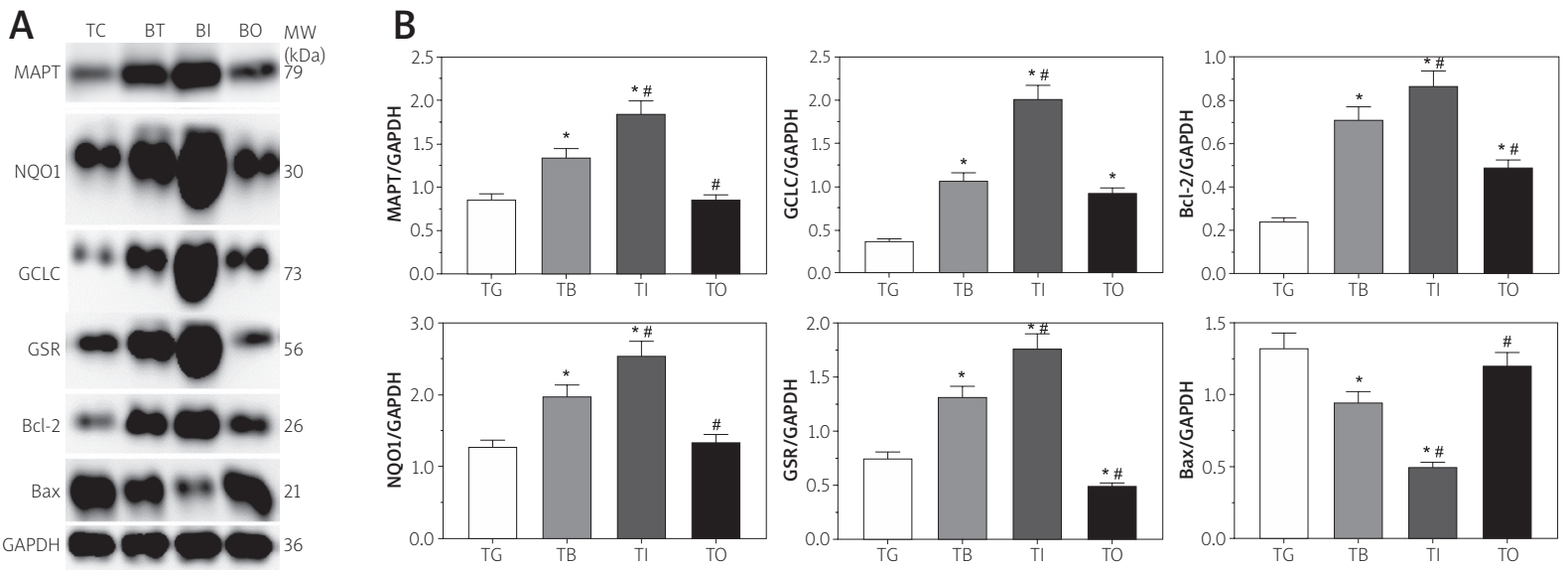

Fig. 5. Detection of glutathione synthesis and cellular apoptosis related enzymes expression in the mice model using Western blotting analysis. A) Expression of glutathione synthesis and cellular apoptosis related enzymes. B) Quantitative analysis of each target proteins. * $p<0.05$ compared with the control group, ${ }^{\#} p<0.05$ compared with the bexarotene treatment group. Each test was repeated independently for three times. One-way ANOVA was used to compare the difference between groups.

\section{Detection of expression of the Nrf2/HO-1 signalling pathway in cell and mouse models}

As shown in Figures 6 and 7, the expression of the Nrf2/HO-1 signalling pathway in cell and mouse models were detected using western blotting analysis. Briefly, the ratio of $\mathrm{p}-\mathrm{MAPK} / \mathrm{MAPK}$ in TC, BT, BI and BO group of $\mathrm{H} 19-7$ cells was $2.28 \pm 0.19,1.66 \pm 0.14$,
$0.83 \pm 0.07$ and $2.18 \pm 0.18$, respectively, and it was $0.80 \pm 0.07,0.61 \pm 0.05,0.24 \pm 0.02$ and $0.78 \pm 0.07$ in TG, TB, TI and TO mice, respectively. The expression of AP-1 in each group of cells was $1.14 \pm 0.10,1.70$ $\pm 0.14,2.11 \pm 0.18$ and $1.32 \pm 0.11$, respectively, and it was $1.34 \pm 0.11,1.46 \pm 0.12,1.75 \pm 0.15$ and $1.09 \pm 0.09$ in each group of mice, respectively. The expression of Kelch-like ECH-associated protein 1 (Keap1) in each group of cells was $1.97 \pm 0.16,1.71 \pm 0.14,0.62 \pm 0.05$
A

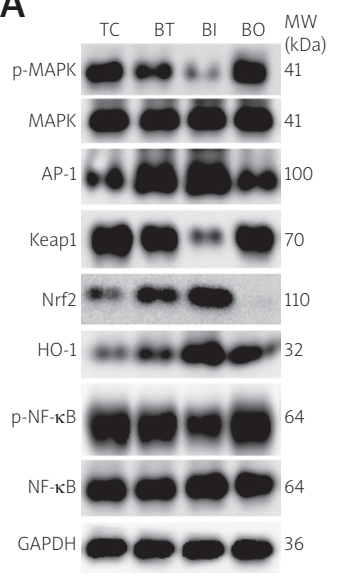

B
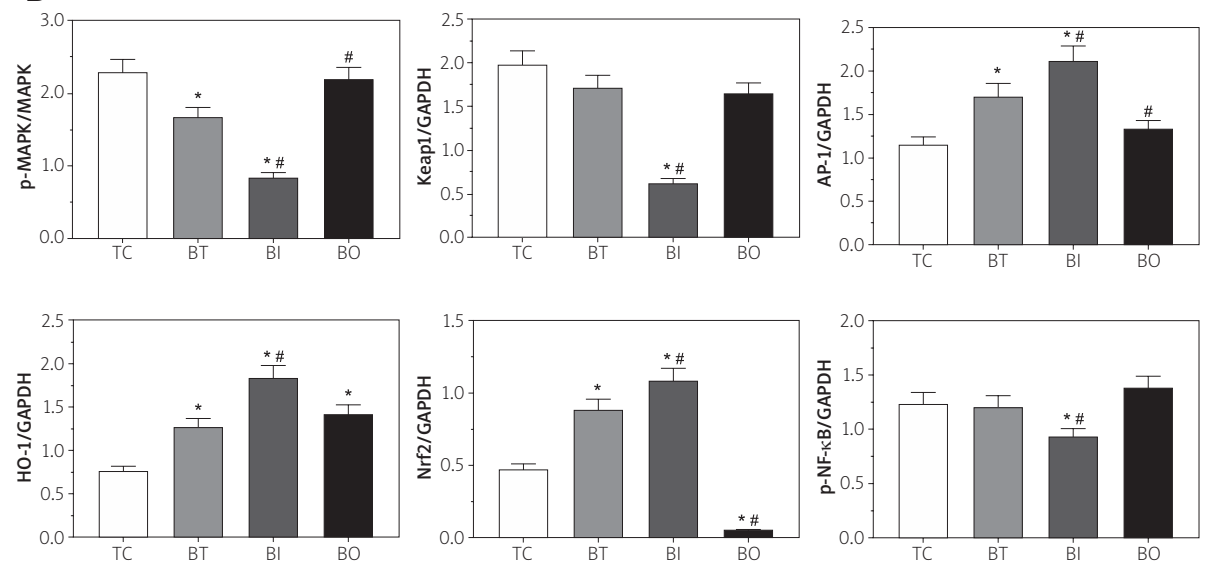

Fig. 6. Detection of activation of Nrf2/HO-1 signalling pathway in $\mathrm{H} 19-7$ cells using Western blotting analysis. A) Expression of glutathione synthesis and cellular apoptosis related enzymes. B) Quantitative analysis of each target proteins. ${ }^{*} p<0.05$ compared with the control group, ${ }^{*} p<0.05$ compared with the bexarotene treatment group. Each experiment was repeated independently for three times. One-way ANOVA was used to compare the difference between groups. 

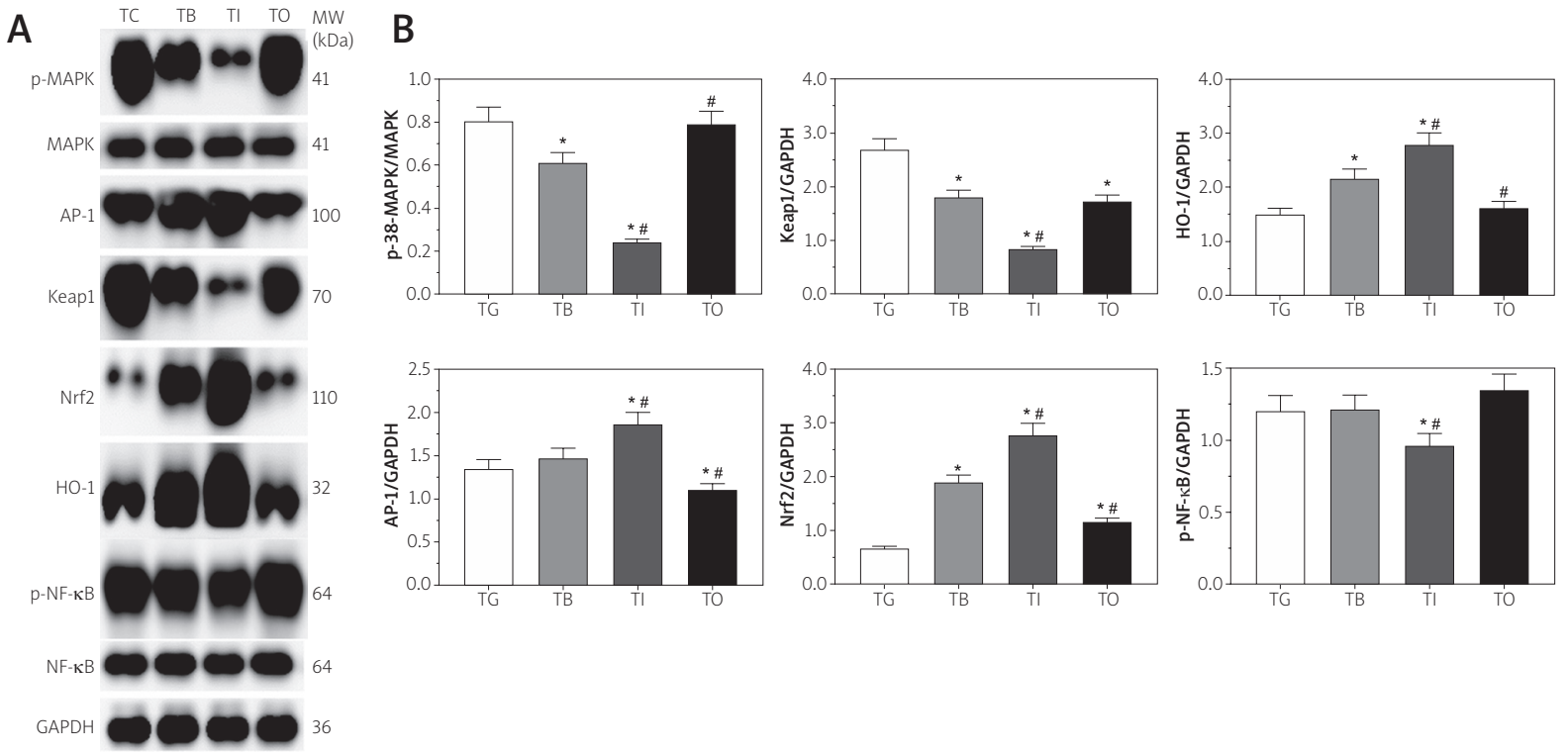

Fig. 7. Detection of activation of Nrf2/HO-1 signalling pathway in the mice model using Western blotting analysis. A) Expression of glutathione synthesis and cellular apoptosis related enzymes. B) Quantitative analysis of each target proteins. ${ }^{*} p<0.05$ compared with the control group, ${ }^{\#} p<0.05$ compared with the bexarotene treatment group. Each experiment was repeated independently for three times. One-way ANOVA was used to compare the difference between groups.

and $1.64 \pm 0.14$, respectively, and it was $2.67 \pm 0.22$, $1.78 \pm 0.15,0.82 \pm 0.01$ and $1.70 \pm 0.14$ in each group of mice, respectively. The expression of Nrf2 in each group of cells was $0.47 \pm 0.04,0.88 \pm 0.07,1.08 \pm 0.09$ and $0.05 \pm 0.01$, respectively, and it was $0.65 \pm 0.05$, $1.87 \pm 0.16,2.75 \pm 0.23$ and $1.13 \pm 0.09$ in each group of mice, respectively. The expression of HO-1 in each group of cells was $0.75 \pm 0.06,1.26 \pm 0.11,1.82 \pm 0.15$ and $1.40 \pm 0.12$, respectively, and it was $1.49 \pm 0.12$, $2.15 \pm 0.18,2.78 \pm 0.23$ and $1.60 \pm 0.13$ in each group of mice, respectively. The ratio of $\mathrm{p}-\mathrm{NF}-\kappa \mathrm{B} / \mathrm{NF}-\kappa \mathrm{B}$ in each group of cells was $1.24 \pm 0.10,1.21 \pm 0.10,0.93 \pm 0.08$ and $1.38 \pm 0.11$, respectively, and it was $1.20 \pm 0.10$, $1.21 \pm 0.10,0.96 \pm 0.08$ and $1.35 \pm 0.11$ in corresponding mice. These results show that the cellular protective effect of bexarotene treatment combined with overexpression of MAPT might be mediated through the p-38 MAPK/Nrf2/HO-1 signalling pathway.

\section{Detection of oxidative stress related cytokines in cultured medium and serum samples}

As shown in Figures 8 and 9, the concentration of oxidative stress-related cytokines in cultured medium and serum samples was detected using
ELISA. The reactive oxygen species (ROS) ratio in TC, $\mathrm{BT}, \mathrm{BI}$ and $\mathrm{BO}$ groups of culture media from $\mathrm{H} 19-7$ cells was $1.00 \pm 0.09,0.81 \pm 0.07,0.53 \pm 0.03$ and 0.95 \pm 0.07 , respectively and it was $1.00 \pm 0.08,0.84 \pm 0.06$, $0.62 \pm 0.04$ and $1.02 \pm 0.11$ in TG, TB, TI and TO groups of serum samples from mice, respectively. The nitric oxide (NO) ratio in each group of culture media from cells was $1.00 \pm 0.07,1.24 \pm 0.12,1.61 \pm 0.14$ and 1.07 \pm 0.09 , respectively, and it was $1.00 \pm 0.08,1.36 \pm 0.14$, $1.72 \pm 0.15$ and $1.12 \pm 0.11$ in each group of serum samples from mice, respectively. The ratio of $\mathrm{H}_{2} \mathrm{O}_{2}$ in each group of culture media from cells was 1.00 $\pm 0.07,0.83 \pm 0.05,0.51 \pm 0.03$ and $0.92 \pm 0.07$, respectively, and it was $1.00 \pm 0.08,0.86 \pm 0.06,0.62 \pm 0.06$ and $0.99 \pm 0.07$ in each group of serum samples from mice, respectively. The ratio of reduced glutathione/ oxidized glutathione (GSH/GSSG) in each group of culture media from cells was $0.42 \pm 0.03,0.71 \pm 0.07$, $1.06 \pm 0.09$ and $0.54 \pm 0.03$, respectively, and it was $0.53 \pm 0.04,0.86 \pm 0.06,1.17 \pm 0.12$ and $0.72 \pm 0.06$ in each group of serum samples from mice, respectively.

\section{Discussion}

Traumatic brain injury is the leading cause of mortality and morbidity worldwide, particularly in 
A

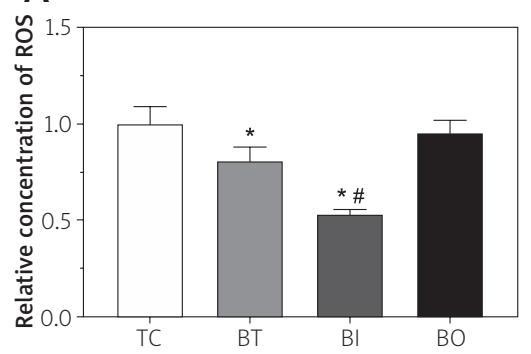

C

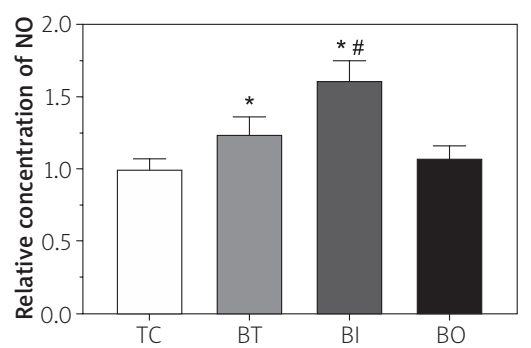

B

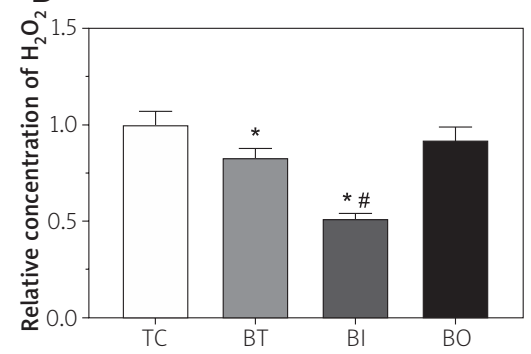

D

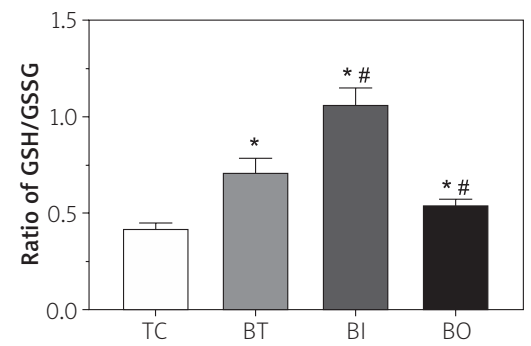

Fig. 8. Relative concentration of oxidative response related cytokines in the cultured medium of H19-7 cells. A) Relative concentration of ROS in the cultured medium of H19-7 cells in each group. B) Relative concentration of $\mathrm{H}_{2} \mathrm{O}_{2}$ in the cultured medium of $\mathrm{H} 19-7$ cells in each group. C) Relative concentration of NO in the cultured medium of H19-7 cells in each group. D) Ratio of GSH/GSSG in the cultured medium of H19-7 cells in each group. ${ }^{*} p<0.05$ compared with the control group, ${ }^{*} p<0.05$ compared with the bexarotene treatment group. Each experiment was repeated independently for three times. One-way ANOVA was used to compare the difference between groups.
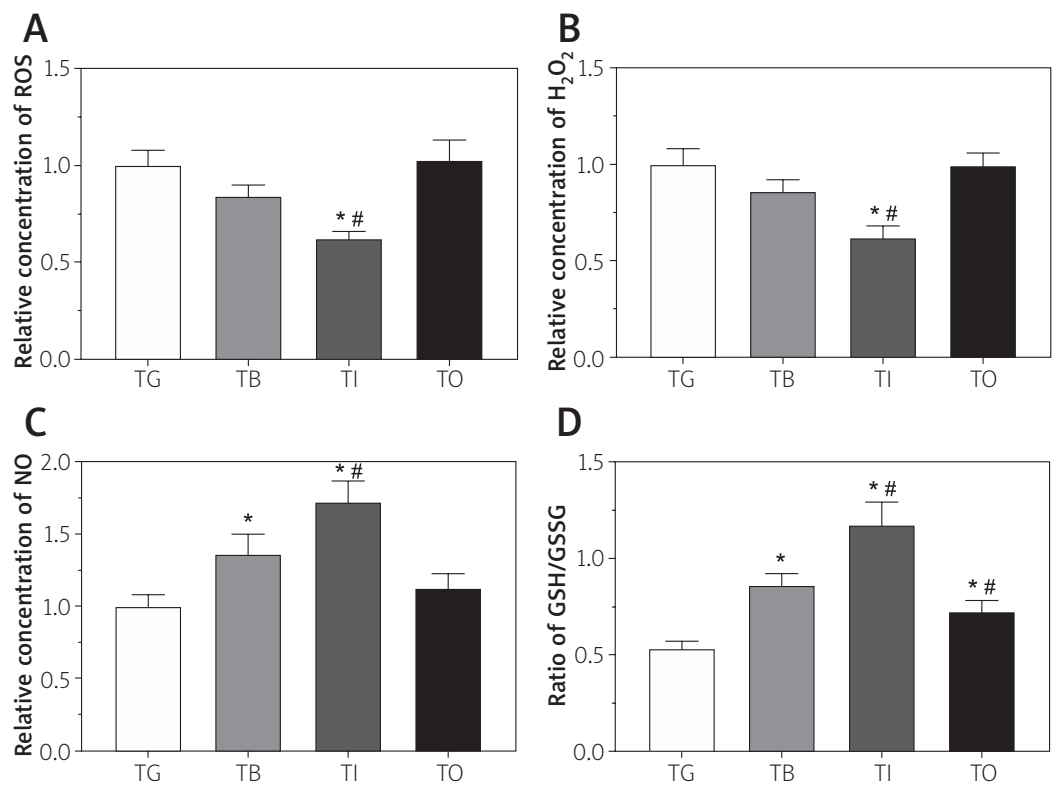

Fig. 9. Relative concentration of oxidative response related cytokines in a serum sample of the mice model. A) Relative concentration of ROS in a serum sample of the mice model in each group. B) Relative concentration of $\mathrm{H}_{2} \mathrm{O}_{2}$ in a serum sample of the mice model in each group. C) Relative concentration of NO in a serum sample of the mice model in each group. D) Ratio of GSH/GSSG in a serum sample of the mice model in each group. ${ }^{*} p<0.05$ compared with the control group, ${ }^{*} p<0.05$ compared with the bexarotene treatment group. Each experiment was repeated independently for three times. One-way ANOVA was used to compare the difference between groups. 
rich countries [14]. Bexarotene is a selective agonist of RXR which has been approved for the treatment of cancer by Food and Drug Administration (FDA) [3], and recent studies observed that bexarotene also exerts a protective role in the treatment of central nervous system (CNS) diseases [33]. However, effective treatments for TBI are still lacking. MAPT is a subtype of the microtubule-associated protein (MAP) family and was first identified in 1975 by Weignarten et al. as a responsible protein for microtubule assembly [28]. A previous study found that aggregation of MAPT is correlated with the progression of Alzheimer's disease, and abnormal MAPT expression was observed in these patients [21]. A recent study found that MAPT is involved in the neurodegeneration process after TBI via regulation of the chronic inflammation process [32]. NQO1 is an enzyme that catalyses electron reduction using $\mathrm{NAD}(\mathrm{P}) \mathrm{H}$ as an electron donor via an FAD-dependent mechanism, and expression of $\mathrm{NQO1}$ is inducible and regulated by Nrf2 and Keap1 [5], which plays an important role in the response to oxidative stress. A previous study has shown that induction or knockdown of NQO1 is closely related with decreased or increased levels of oxidative stress [22]. GCLC, also known as the glutamate-cysteine ligase catalytic subunit gene, encodes the expression of glutamate cysteine ligase enzyme and regulates the first rate-limiting enzyme of cellular glutathione (GSH) biosynthetic pathway [23]. A previous study found that GCLC contains the active site, responsible for ATP-dependent bond formation between the amino group of cysteine and the $\gamma$-carboxyl group of glutamate. GSH also inhibits the activity of GCL by competing with glutamate at the active site of GCLC [8]. Furthermore, under oxidative stress status, production of ROS activates the Nrf2 pathway, resulting in an increased expression of GCLC, which protects cells against oxidative stress [30]. Glutathione reductase (GSR) is a member of the subfamily of flavoprotein oxidoreductases and catalyses the transformation of GSSG into GSH in an NADPH-dependent manner [24]. Thus, GSR plays an important role in resisting oxidative stress and maintaining cellular homeostasis through regulation of GSH/GSSG balance [10]. Aside from oxidative stress induced by damage in neurological cells, TBI also induces apoptosis, and mitochondria plays an important role in regulation of cellular survival and apoptotic death, including loss of mitochondrial transmembrane potential, changes in cellular oxidation-reduction status and regulation of $\mathrm{BCl}-2$ family proteins [9]. $\mathrm{Bcl}-2$ is the primary antiapoptotic member of the $\mathrm{Bcl}-2$ family of proteins, and regulates membrane integrity and release of cytochrome C. Bax is the primary pro-apoptotic member, located in the cytoplasm and translocating into the mitochondria in response to death stimuli [15]. The balance between $\mathrm{BCl}-2$ and Bax determines the survival or apoptotic process of cells [16]. An increased expression of $\mathrm{BCl}-2$ and decreased expression of $\mathrm{Bax}$ leads to cell survival, indicating that overexpression of MAPT enhanced the protective effect of bexarotene in treatment of a TBI model via promoting the survival of neuronal cells.

Generation of ROS is a characteristic pathological presentation after TBI, which leads to apoptosis and death of neuronal cells. Under normal conditions, Nrf2 binds with the ubiquitin ligase systems via Keap1, promoting the continuous ubiquitination and degradation of Nrf2 [6]. However, under oxidative stress condition, radicals attack the cysteine residues of Keap1, leading to the dissociation and activation of Nrf2 [25]. Activation of Nrf2 increases the transcription of a wide array of downstream cytoprotective genes, including hemeoxygenase-1 (HO-1) and $\mathrm{NAD}(\mathrm{P}) \mathrm{H}$ :quinone oxidoreductase 1 (NQO1) [16]. Among multiple factors, Nrf2 exerts a significant neuroprotective role in $\mathrm{TBI}$ and neurodegenerative disorders, and inhibition of Nrf2 expression exacerbates the damage induced by TBI according to a previous study [7]. In contrast, the expression of NF- $\mathrm{kB}$ in neurons after TBI causes long-term inflammatory activation [18], while inhibition of NF-kB expression promotes neuronal cell death, worsens neurological outcomes and increases the posttraumatic mortality rate [17]. A recent study also found that an induced expression of Nrf2 promotes the expression of the anti-apoptotic BclXL protein and downregulation of Bax and other proapoptotic proteins via inhibition of NF-KB expression [11]. Moreover, cellular damage signals induce the activation of the MAPK signalling pathway, leading to the release of pro-inflammatory factors that aggravate the damage to neuronal cells in response to TBI via interaction with toll-like receptors [2]. Using an Alzheimer's mouse model, researchers found that inhibition of the MAPK expression induced the accumulation of amyloid- $\beta$, enhancing neurodegeneration symptoms in the mouse model [31]. A decreased expression of NF-KB and MAPK was observed after treatment of bexarotene and was increased after overexpression of MAPT, indicat- 
ing that overexpression of MAPT might enhance the therapeutic effects of bexarotene on TBI via alleviating the oxidative stress process.

\section{Disclosure}

\section{The authors declare no conflict of interest.}

\section{References}

1. Barker-Collo S, Theadom A, Jones K, Starkey N, Kahan M, Feigin $V$. Depression and anxiety across the first 4 years after mild traumatic brain injury: Findings from a community-based study. Brain Inj 2018; 32: 1651-1658.

2. Bergold PJ. Treatment of traumatic brain injury with antiinflammatory drugs. Exp Neurol 2016; 275: 367-380.

3. Cramer PE, Cirrito JR, Wesson DW, Karlo JC, Zinn AE, Casali BT, Restivo JL, Goebel WD, James MJ, Brunden KR, Wilson DA, Landreth GE. ApoE-directed therapeutics rapidly clear beta-amyloid and reverse deficits in AD mouse models. Science 2012; 335: 1503-1506.

4. Dheer Y, Chitranshi N, Gupta V, Abbasi M, Mirzaei M, You Y, Chung R, Graham SL, Gupta V. Bexarotene modulates retinoid$X$-receptor expression and is protective against neurotoxic endoplasmic reticulum stress response and apoptotic pathway activation. Mol Neurobiol 2018; 55: 9043-9056.

5. Dinkova-Kostova AT, Talalay P. NAD(P)H:quinone acceptor oxidoreductase 1 (NQO1), a multifunctional antioxidant enzyme and exceptionally versatile cytoprotector. Arch Biochem Biophys 2010; 501: 116-123.

6. Dinkova-Kostova AT, Abramov AY. The emerging role of Nrf2 in mitochondrial function. Free Radic Biol Med 2015; 88: 179-188.

7. Dong W, Yang B, Wang L, Guo X, Zhang M, Jiang Z, Fu J, Pi J, Guan D, Zhao R. Curcumin plays neuroprotective roles against traumatic brain injury partly via Nrf2 signaling. Toxicol Appl Pharmacol 2018; 346: 28-36.

8. Franklin CC, Backos DS, Mohar I, White CC, Forman HJ, Kavanagh TJ. Structure, function, and post-translational regulation of the catalytic and modifier subunits of glutamate cysteine ligase. Mol Aspects Med 2009; 30: 86-98.

9. Green DR, Reed JC. Mitochondria and apoptosis. Science 1998; 281: 1309-1312.

10. Ji M, Barnwell CV, Grunden AM. Characterization of recombinant glutathione reductase from the psychrophilic Antarctic bacterium Colwellia psychrerythraea. Extremophiles 2015; 19: 863-874.

11. Liu Y, Yang H, Wen Y, Li B, Zhao Y, Xing J, Zhang M, Chen Y. Nrf2 inhibits periodontal ligament stem cell apoptosis under excessive oxidative stress. Int J Mol Sci 2017; 18: E1076.

12. Livak KJ, Schmittgen TD. Analysis of relative gene expression data using real-time quantitative PCR and the 2(-Delta Delta C(T)) method. Methods 2001; 25: 402-408.

13. Ma Y, Liu W, Wang Y, Chao X, Qu Y, Wang K, Fei Z. VEGF protects rat cortical neurons from mechanical trauma injury induced apoptosis via the MEK/ERK pathway. Brain Res Bull 2011; 86: 441-446.

14. Maas AIR, Menon DK, Adelson PD, Andelic N, Bell MJ, Belli A, Bragge P, Brazinova A, Büki A, Chesnut RM, Citerio G, Coburn M,
Cooper DJ, Crowder AT, Czeiter E, Czosnyka M, Diaz-Arrastia R, Dreier JP, Duhaime AC, Ercole A, van Essen TA, Feigin VL, Gao G, Giacino J, Gonzalez-Lara LE, Gruen RL, Gupta D, Hartings JA, Hill S, Jiang JY, Ketharanathan N, Kompanje EJO, Lanyon L, Laureys S, Lecky F, Levin $\mathrm{H}$, Lingsma HF, Maegele $M$, Majdan $M$, Manley G, Marsteller J, Mascia L, McFadyen C, Mondello S, Newcombe V, Palotie A, Parizel PM, Peul W, Piercy J, Polinder S, Puybasset L, Rasmussen TE, Rossaint R, Smielewski P, Söderberg J, Stanworth SJ, Stein MB, von Steinbüchel N, Stewart W, Steyerberg EW, Stocchetti N, Synnot A, Te Ao B, Tenovuo O, Thea$\operatorname{dom}$ A, Tibboel D, Videtta W, Wang KKW, Williams WH, Wilson L, Yaffe K; InTBIR Participants and Investigators. Traumatic brain injury: integrated approaches to improve prevention, clinical care, and research. Lancet Neurol 2017; 16: 987-1048.

15. Martin S, Toquet C, Oliver L, Cartron PF, Perrin P, Meflah K, Cuillère $P$, Vallette FM. Expression of bcl-2, bax and bcl-xl in human gliomas: a re-appraisal. J Neurooncol 2001; 52: 129-139.

16. Marrot L, Jones C, Perez P, Meunier JR. The significance of Nrf2 pathway in (photo)-oxidative stress response in melanocytes and keratinocytes of the human epidermis. Pigment Cell Melanoma Res 2008; 21: 79-88.

17. Mettang M, Reichel SN, Lattke M, Palmer A, Abaei A, Rasche V, Huber-Lang M, Baumann B, Wirth T. IKK2/NF-kappaB signaling protects neurons after traumatic brain injury. FASEB J 2018; 32: 1916-1932.

18. Nonaka M, Chen XH, Pierce JE, Leoni MJ, McIntosh TK, Wolf JA, Smith DH. Prolonged activation of NF-kappaB following traumatic brain injury in rats. J Neurotrauma 1999; 16: 1023-1034.

19. Neselius S, Brisby H, Theodorsson A, Blennow K, Zetterberg H, Marcusson J. CSF-biomarkers in olympic boxing: diagnosis and effects of repetitive head trauma. PLoS One 2012; 7: e33606.

20. Neselius S, Zetterberg H, Blennow K, Randall J, Wilson D, Marcusson J, Brisby H. Olympic boxing is associated with elevated levels of the neuronal protein tau in plasma. Brain Inj 2013; 27 : 425-433.

21. Pîrşcoveanu DFV, Pirici I, Tudorică V, Bălşeanu TA, Albu VC, Bondari S, Bumbea AM, Pîrşcoveanu M. Tau protein in neurodegenerative diseases - a review. Rom J Morphol Embryol 2017; 58: 1141-1150.

22. Ross D, Kepa JK, Winski SL, Beall HD, Anwar A, Siegel D. NAD(P) $\mathrm{H}$ :quinone oxidoreductase 1 (NQO1): chemoprotection, bioactivation, gene regulation and genetic polymorphisms. Chem Biol Interact 2000; 129: 77-97.

23. Saragih H, Zilian E, Jaimes Y, Paine A, Figueiredo C, Eiz-Vesper B, Blasczyk R, Larmann J, Theilmeier G, Burg-Roderfeld M, AndreiSelmer LC, Becker JU, Santoso S, Immenschuh S. PECAM-1-dependent heme oxygenase-1 regulation via an Nrf2-mediated pathway in endothelial cells. Thromb Haemost 2014; 111: 1077-1088.

24. Sikanyika M, Aragão D, McDevitt CA, Maher MJ. The structure and activity of the glutathione reductase from Streptococcus pneumoniae. Acta Crystallogr F Struct Biol Commun 2019; 75: 54-61.

25. Surh YJ, Kundu JK, Na HK. Nrf2 as a master redox switch in turning on the cellular signaling involved in the induction of cytoprotective genes by some chemopreventive phytochemicals. Planta Med 2008; 74: 1526-1539.

26. Taylor CA, Bell JM, Breiding MJ, Xu L. Traumatic brain injuryrelated emergency department visits, hospitalizations, and 
deaths - United States, 2007 and 2013. MMWR Surveill Summ 2017; 66: 1-16.

27. Trojanowski JQ, Schuck T, Schmidt ML, Lee VM. Distribution of tau proteins in the normal human central and peripheral nervous system. J Histochem Cytochem 1989; 37: 209-215.

28. Weingarten MD, Lockwood AH, Hwo SY, Kirschner MW. A protein factor essential for microtubule assembly. Proc Natl Acad Sci U S A 1975; 72: 1858-1862.

29. Xu X, Yin D, Ren H, Gao W, Li F, Sun D, Wu Y, Zhou S, Lyu L, Yang M, Xiong J, Han L, Jiang R, Zhang J. Selective NLRP3 inflammasome inhibitor reduces neuroinflammation and improves long-term neurological outcomes in a murine model of traumatic brain injury. Neurobiol Dis 2018; 117: 15-27.

30. Zhang Y, Song M, Rui X, Pu S, Li Y, Li C. Supplemental dietary phytosterin protects against 4-nitrophenol-induced oxidative stress and apoptosis in rat testes. Toxicol Rep 2015; 2: 664-676.

31. Zhao C, Zhang H, Li H, Lv C, Liu X, Li Z, Xin W, Wang Y, Zhang W. Geniposide ameliorates cognitive defcits by attenuating the cholinergic defect and amyloidosis in middle-aged Alzheimer model mice. Neuropharmacology 2017; 116: 18-29.

32. Zhao J, Xu C, Cao H, Zhang L, Wang X, Chen S. Identification of target genes in neuroinflammation and neurodegeneration after traumatic brain injury in rats. PeerJ 2019; 7: e8324.

33. Zhong J, Cheng C, Liu H, Huang Z, Wu Y, Teng Z, He J, Zhang H, Wu J, Cao F, Jiang L, Sun X. Bexarotene protects against traumatic brain injury in mice partially through apolipoprotein $\mathrm{E}$. Neuroscience 2017; 343: 434-448. 